\title{
Effect of Material Damage on Autofrettage of Thick-Walled Cylinder
}

\author{
Gennadiy Lvov, Olga Kostromitskaya* \\ Department of Dynamics and Strength of Machines, Kharkov Polytechnic University, Kharkov, 61002, Ukraine \\ *Corresponding Author: kostrom_olga@mail.ru
}

Copyright $@ 2014$ Horizon Research Publishing All rights reserved.

\begin{abstract}
Continuum damage mechanics approach has been used for analysis of the autofrettage process. General set of government equations of elastic-plastic bodies is derived by using the effective stress concept. Strain damage coupling constitutive equations are developed on the base of plasticity relationships with bilinear kinematic hardening and kinetic law of ductile damage evolution. Damage of the material leads to decrease of Young's modulus after plastic deforming that affects on the unloading stage of autofrettage. Influence of material damage on residual hoop stress was considered by the example of autofrettaged thick-walled cylinder. Numerical results were obtained by finite-element method for plane-strain case. These results showed that material damage modifies the residual stress distributions and reduced residual hoop stress value on bore area.
\end{abstract}

Keywords Autofrettage, Damage, Residual Stresses, Thick-Walled Cylinder

\section{Introduction}

For many high pressure construction units working at cyclic high loading the autofrettage method is most frequently used for increase of fatigue resistance. Autofrettage is operating process in which construction units are loaded by high technological load so that part of the unit becomes plastic. When the technological load is removed favorable residual stresses remain in the construction unit.

Autofrettage is most spread for thick-walled cylinders which are loaded by internal pressure in operating process. Hoop tensile stresses on bore area are maximal in service conditions of cylinders. The autofrettage process allows creating compressive residual stresses on bore area. Superposition of residual stresses with stresses arising in the process of exploitation results to decrease of maximal stresses under working conditions.

Theoretical and experimental investigations in this area of solid mechanics attract considerable interest because of large applied sense of the autofrettage. Investigations of autofrettage on the base of perfect plasticity model [1] and isotropic hardening model [2,3] made possible to estimate the level of residual stresses at first approximation.

Process of residual stresses generation in thick-walled cylinder was investigated taking into consideration temperature strains in paper [4]. Model Prandtl-Reuss was used for plastic yielding of isotropic material.

Anisotropic hardening is taken into account for more precise modeling of unloading under autofrettage. Papers $[5,6]$ are devoted to modeling of the autofrettage procedure for thick-walled cylinder incorporating the Bauschinger effect [7]. This effect consists of a reduction of the yield stress in compression as a result of a previous tensile yielding. The influence of the Bauschinger effect results the decrease of compressive residual stresses on inner bore in comparison with isotropic hardening and decreases the beneficial autofrettage effect. Redistribution of residual stresses in consequence of removing material from the inside bore was considered by Parker et al [6]. Experimental procedure of determination of residual stresses by the Sach's method is considered. Comparison theoretical and experimental results showed significant difference in residual stresses on inner bore.

The Bauschinger effect was explored at the analysis [8] of compound pipes containing an autofrettaged layer. The author of the article suggested using the autofrettaged thick-walled pipe as part of whole construction.

The numerical procedure for calculate of autofrettage is offered for open-end tubes [9]. Such boundary conditions conform to generalized plane strain (constant axial strain with zero net axial force). Also in this article distributions of residual stresses were calculated for von Mises criterion for plane strain and closed-end cases. The numerical results showed that the Bauschinger effect substantially influences on residual stress profiles.

Autofrettage of variable thickness thick-walled cylinder with technological bandages was considered [10] on the basis of a variation formulation for the elastic-plastic contact problem. The numerical solution was obtained by the finite element method with the material model which takes kinematical hardening and the ideal Bauschinger effect into account. 
Experimental Bauschinger effect date of high strength steel were used in [11] for calculating autofretage of thick-walled cylinder. Analytical and finite-element analysis found that residual stress incorporating the Bauschinger effect at inner bore was smaller than for isotropic hardening.

The autofrettage model based on the real tensile-compressive curve of material is proposed in [12]. Analytic solution for residual stresses was obtained with von Mises yield criterion for incompressible material.

Various researches are performed on optimization of autofrettage processes on different criteria [13-16]. Possibilities of the autofrettage spherical vessels are investigated in [17] using Prandtl-Reuss flow rule.

In the process of autofrettage considerable plastic strains are developed in the material. Plastic deformation of the material is accompanied by development of micro damage which can decreases the beneficial autofrettage effect and even results in the reduction of strength properties of structural elements. Damage of the material at plastic deformation shows up even at the single loading as the decrease of Young's modulus. Tendency to increasing the effect of autofrettage results in complication of the programs of loading, use of repeated of mechanical and thermal cycles [18]. For such autofrettage processes damage of the material has essential value.

These circumstances cause a necessity to investigate the process of autofrettage taking into account the effects of material damage at plastic deformation. In this work such research is executed on the background of continuum damage mechanics [19].

\section{Plastic-Damage Governing Equations}

Consider the geometrically linear formulation problem of the residual stresses remained after elastic-plastic deformation and unloading of solid body. Represent in the system of curvilinear coordinates a body occupying in the initial state volume $V$ with a boundary $S_{\mathrm{U}} \cup S_{P}$, where $S_{\mathrm{U}}$ part of surface which displacement is set on, $S_{P}$ - is part of surface with the set of external loadings. Loading and unloading of body at autofrettage is considered as unified process in time. The program of external load change at autofrettage is dividing into a series of infinitesimal increment of surface $d P_{i}$ and volume $d X_{\mathrm{i}}$ forces. Rates of stresses $\dot{\sigma}_{i j}$, strains $\dot{\varepsilon}_{i j}$ and displacements $\dot{\mathrm{u}}_{\mathrm{i}}$ on every stage of time dt must satisfy to the system of equations

$$
\begin{aligned}
& \nabla_{i} \dot{\sigma}_{i j}+\dot{X}_{j}=0 \\
& \dot{\varepsilon}_{i j}=1 / 2\left(\nabla_{i} \dot{u}_{j}+\nabla_{j} \dot{u}_{i}\right) \quad x \in V \\
& \dot{\varepsilon}_{i j}=\left(C_{i j k l}^{e}+C_{i j k l}^{p}\right) \dot{\tilde{\sigma}}_{k l}
\end{aligned}
$$

Here $\nabla_{i}$ - is sign of covariant derivation in the chosen system of coordinates, $C_{i j k l}^{e}$ - is tensor of elastic constants,
$C_{i j k l}^{p}$ - is tensor of plastic compliance values of which are determined on every time step by the chosen plasticity model.

Principle of equivalent stress was used in these relationships for the application of material damage. According to this principle any stress in constitutive equations for the damaged material can be used the same way as for undamaged material by replacement of Cauchy stress tensor on effective stress tensor $\tilde{\sigma}_{i j}[19]$ :

$$
\tilde{\sigma}_{i j}=\frac{\sigma_{i j}}{(1-D)},
$$

where $\mathrm{D}$ - is damage variable $(0 \leq D \leq 1)$ which is interpreted as relative area of micro cavities. Scalar variable $\mathrm{D}$ is equal 0 for undamaged material and 1 for fully broken material when the damages occupy whole plane.

The processes of deformation at autofrettage are very complex. The changes of stress sign and development of plastic strains are possible at pressure removing. Constitutive equations must adequately describe quantitative and quality features of plastic deformation process, in particular, anisotropic hardening and the Bauschinger effect.

The plasticity theory with the kinematic hardening satisfies such requirements $[18,5,20]$. Equation of yield surface is accepted in modified form:

$$
f \equiv 3 / 2\left(\tilde{s}_{i j}-c \varepsilon_{i j}^{p}\right)\left(\tilde{s}_{i j}-c \varepsilon_{i j}^{p}\right)-\sigma_{T}^{2}
$$

where $f$ - equation of yield surface, $\tilde{s}_{i j}$ - deviator of effective stresses, $\varepsilon_{i j}^{p}$ - is plastic strain tensor, $\sigma_{T}$ - yield stress, $c$ - parameter of hardening.

Tensor of plastic compliance for the chosen variant of plasticity model at the active loading

$$
f=0, \quad \frac{\partial f}{\partial \tilde{\sigma}_{i j}} \dot{\tilde{\sigma}}_{i j} \succ 0
$$

looks like:

$$
C_{i j k l}^{p}=\frac{\left(\tilde{s}_{k l}-c \varepsilon_{k l}^{p}\right)}{c\left(\tilde{s}_{m n}-c \varepsilon_{m n}^{p}\right)\left(\tilde{s}_{m n}-c \varepsilon_{m n}^{p}\right)}\left(\tilde{s}_{i j}-c \varepsilon_{i j}^{p}\right)
$$

Plastic strains are not added at the neutral loading and unloading:

$$
C_{i j k l}^{p}=0 ; \quad\left\{\begin{array}{l}
f \prec o \\
f=0 \quad \frac{\partial f}{\partial \tilde{\sigma}_{i j}} \dot{\tilde{\sigma}}_{i j} \leq 0
\end{array}\right.
$$

Constitutive equations must be completed by the kinetic law of damage parameter. For hardening elastic-plastic material the damage parameter rate is set by relationship [19]

$$
\dot{D}=\frac{\sigma_{e q}^{2}}{2 E S(1-D)^{2}} \dot{p}, p \geq p_{D}
$$

where $p_{D}$ - is some limit of equivalent plastic strain after 
which the first damages are appeared, $\dot{p}=\sqrt{\frac{2}{3}\left\{d \dot{\varepsilon}_{i j}^{p}\right\} \cdot\left\{d \dot{\varepsilon}_{i j}^{p}\right\}}$ - rate equivalent plastic strain, $\sigma_{e q}$ - equivalent stress, $S$ - is constant of damage law which is determined experimentally [19].

The system of equations (1) is completed by boundary conditions which are determined by the fixing and loading of body.

$$
\begin{array}{ll}
\dot{P}_{i}=\dot{\sigma}_{i j} \cdot n_{j} & x \in S_{p} \\
\dot{u}_{i}=d u_{i}^{*} & x \in S_{u}
\end{array}
$$

\section{Axially Symmetric Problem}

Strengthening of construction units by autofrettage is most distributed for the thick-walled cylinders and bodies of rotation with a variable thickness. Here the autofrettage problem for infinitely long thick-walled cylinder is considered with the use of the cylindrical system of coordinates.

Geometrical correlations (1) for the rate strains in cylindrical coordinates look like:

$$
\dot{\varepsilon}_{r}=\frac{\partial \dot{u}}{\partial r}, \quad \dot{\varepsilon}_{\theta}=\frac{\dot{u}}{r}
$$

Differential equilibrium equation (1) of axially symmetric problem is written down for the rate stress:

$$
\frac{\partial \dot{\sigma}_{r}}{\partial r}+\frac{\dot{\sigma}_{r}-\dot{\sigma}_{\theta}}{r}=0
$$

Expressions for the rate stress are got by the inversion of constitutive equations in (1):

$$
\begin{gathered}
\dot{\sigma}_{r}=2 \mu(1-D)\left(\dot{\varepsilon}_{r}-\dot{\varepsilon}_{r}^{p}\right)+\lambda \dot{\theta}(1-D) \\
\dot{\sigma}_{\theta}=2 \mu(1-D)\left(\dot{\varepsilon}_{\theta}-\dot{\varepsilon}_{\theta}^{p}\right)+\lambda \dot{\theta}(1-D) \\
\mu=\frac{E}{1+v}, \quad \lambda=\frac{v E}{(1+v)(1-2 v)}, \quad \dot{\theta}=\dot{\varepsilon}_{r}+\dot{\varepsilon}_{\theta}
\end{gathered}
$$

where E- is Young's modulus and $v$ - is Poisson's ratio of material in the initial damaged state. Governing equations (8) of axially symmetric problem are obtained after substitution of (5) and (7) in (6) .

$$
\begin{gathered}
\frac{2-3 v}{1-2 v}(1-D) \frac{\partial^{2} \dot{u}}{\partial r^{2}}+\frac{2-3 v}{1-2 v}\left(\frac{1-D}{r}-D_{r}^{\prime}\right) \frac{\partial \dot{u}}{\partial r}- \\
-\left(\frac{2-3 v}{1-2 v} \cdot \frac{1-D}{r}+\frac{v D_{r}^{\prime}}{1-2 v}\right) \frac{\dot{u}}{r}- \\
-2(1-D)\left(\dot{\varepsilon}_{r}^{p}\right)_{r}^{\prime}-2\left(\frac{1-D}{r}-D_{r}^{\prime}\right) \dot{\varepsilon}_{r}^{p}+ \\
+\frac{2}{r}(1-D) \dot{\varepsilon}_{\theta}^{p}=0
\end{gathered}
$$

Associated flow rule taking into account the criterion of yield (3)

$$
\begin{aligned}
& \dot{\varepsilon}_{r}^{p}=\frac{3}{2 \tilde{\sigma}_{e q}}\left(\tilde{s}_{r}-c \varepsilon_{r}^{p}\right) \dot{p} \\
& \dot{\varepsilon}_{\theta}^{p}=\frac{3}{2 \tilde{\sigma}_{e q}}\left(\tilde{s} \theta-c \varepsilon_{\theta}^{p}\right) \dot{p}
\end{aligned}
$$

where $\tilde{\sigma}_{e q}$ - equivalent effective stress:

$$
\tilde{\sigma}_{e q}=(1 / \sqrt{2}) \sqrt{\left(\tilde{\sigma}_{r}-\tilde{\sigma}_{\theta}\right)^{2}+\tilde{\sigma}_{r}^{2}+\tilde{\sigma}_{\theta}^{2}} .
$$

The qualitative features of damage influence on distributing of residual stress were investigated on the plane strain of infinitely long thick-walled pipe. The numerical study was performed using the ANSYS finite element software. Constitutive equations of plasticity taking into account damage (2) were implemented by user's routine created on the base of the USERPL.f. module.

The calculations are conducted for long thick-walled cylinder of constant thickness with the following sizes: internal radius $a=0.05 \mathrm{~m}$; external radius $b=0.12 \mathrm{~m}$.

The two materials are used for the calculations. One follow the Von Mises yield criteria with the bilinear kinematic hardening. The Bauschinger effect is considered.

The first material has following properties: yield stress, $\sigma_{T}=12.0 \cdot 10^{8} \cdot \mathrm{Pa}$; Young's Modulus, $\mathrm{E}=2.1 \cdot 10^{11} \mathrm{~Pa}$; tangent module of deformation diagram with the bilinear hardening $E_{T}=2.1 \cdot 10^{10} \mathrm{~Pa}$, and Poisson's Ratio $v=0.29$.

The second material takes into account damage. There was used the procedure USERPL.f of ANSYS on the basis of which created custom subroutine for a description of the material. For this material, except for the above-mentioned characteristics, the parameters of damage were used following coefficient $C=1.56 \cdot 10^{10} \mathrm{~Pa}$ in equation of yield surface (3); constant $S=7.0 \cdot 10^{6} \mathrm{~Pa}$ [19] in kinetic law of damage (4).

The finite element model of the thick-walled cylinder with the coordinate system and geometric parameters is shown in Figure 1. A plane model of the cylinder was constructed in ANSYS with two-dimensional axisymmetric PLANE42 elements. Based on the mesh study, the finer mesh was created around the inner radius where maximum stress occurred, as shown in Figure 2. There are 50 elements in radial direction of the cylinder. Since autofrettage at the maximum stresses are located closer to the inner radius of the pipe, it uses the non-uniform finite element mesh, the size of the elements along the radius is not the same. The size of the element belonging to the inner radius of the pipe in two times smaller than the size of the outer radius. The model along the $y$-axis has the size hy $=0.015 \mathrm{~m}$. To simulate a long pipe with such a short model is given the appropriate boundary conditions and sets of coupled degrees of freedom. Displacements $U y=0$ for the nodes lying on the line $y=0$. Also set to the same displacement Uy for all the nodes that belong to the line $\mathrm{y}=$ hy and the same radial displacement 
Ux of the nodes lines $\mathrm{y}=$ hy and $\mathrm{y}=0$.

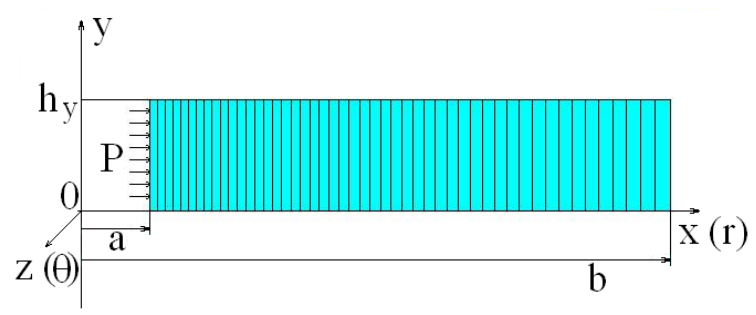

Figure 1. FE model of the thick-walled cylinder

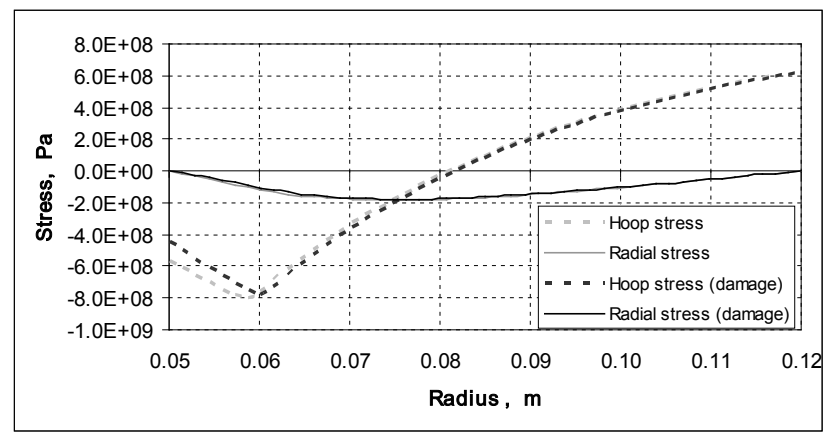

Figure 2. Residual stresses

Load applied in parts - in $\mathrm{n}=7$ steps. For this program is written in the language APDL. The first step of loading $\mathrm{P}_{0}=$ $0.9 \cdot 10^{8} \mathrm{~Pa}$ corresponds to elastic deformation of the cylinder, the hoop stresses in this case are slightly less than the yield stress. We solve the problem of determining the stress-strain state of the pipe under internal pressure. Further to the initial value $\mathrm{P}_{0}$ added pressure increment $\Delta \mathrm{P}=\left(\mathrm{P}_{\max }-\mathrm{P}_{0}\right) /(\mathrm{n}-1): \mathrm{P}_{\mathrm{i}}=$ $\mathrm{P}_{0}+\mathrm{i} \cdot \Delta \mathrm{P}, \quad i=\overline{2, n}$. Deformation in these steps is elastic-plastic.

Seven values of internal pressure, $\mathrm{P}_{\max }$, were analyzed

$\mathrm{P}_{\text {max }}=1.0 \cdot 10^{9}, 1.2 \cdot 10^{9}, 1.3 \cdot 10^{9}, 1.5 \cdot 10^{9}, 1.6 \cdot 10^{9}, 1.7 \cdot 10^{9}$, $2.0 \cdot 10^{9}(\mathrm{~Pa})$.

At each step, we solve the problem of determining the stress-strain state. When the problem is solved for $\mathrm{P}_{\max }$, begins load reduction during six steps and the pressure is released to zero: $\mathrm{P}_{\mathrm{i}}=\mathrm{P}_{\max }-\mathrm{i} \cdot \Delta \mathrm{P}, i=\overline{n+1,(2 n-1)}$. At each step, we solve the problem of unloading and determined the residual stresses and strains. In Figures 3-5 shows the results of solving the problems of unloading for some of the values $\mathrm{P}_{\max }$.

The autofrettage process was simulated by the incremental adding of bore pressure $P$ (Figure 1) from a zero to the maximal value of $P_{\max }$. Then bore pressure incrementally removed to the zero.

Residual hoop and radial stress distributions are presented on the thickness of cylinder after the autofrettage process with pressure $P_{\max }=1.5 \cdot 10^{9} \mathrm{~Pa}$ (Figure 2). Residual stresses are represented without taking into account damage material and - taking into account damage.

Residual hoop stresses profiles for the different values of the autofrettage pressure $\mathrm{P}_{\max }$ are illustrated on a Figure 3 These profiles relate to governing equation of material with damage and the Bauschinger effect.

Distributing of damage parameter is shown on a Figure 4.

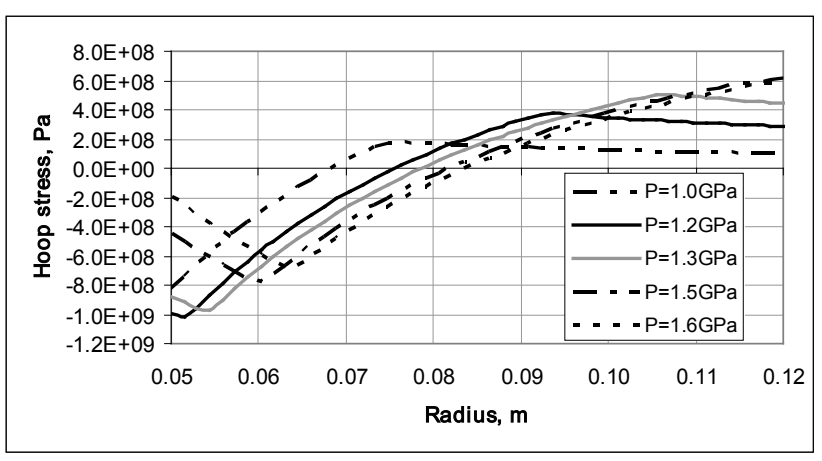

Figure 3. Residual stresses for material with damage

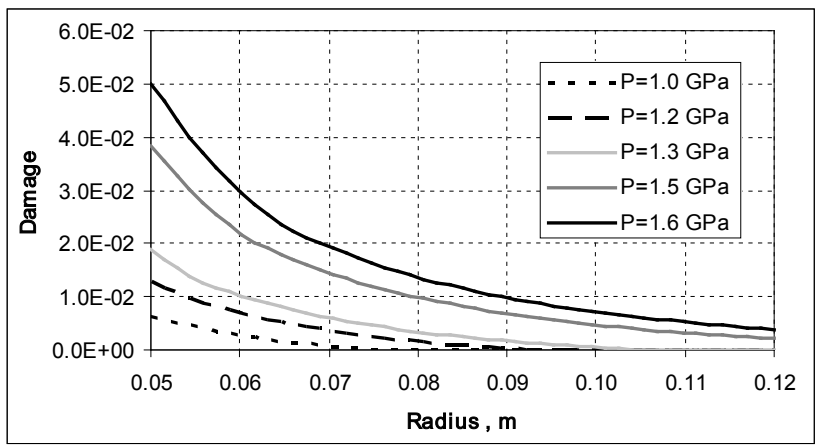

Figure 4. Damage parameter D

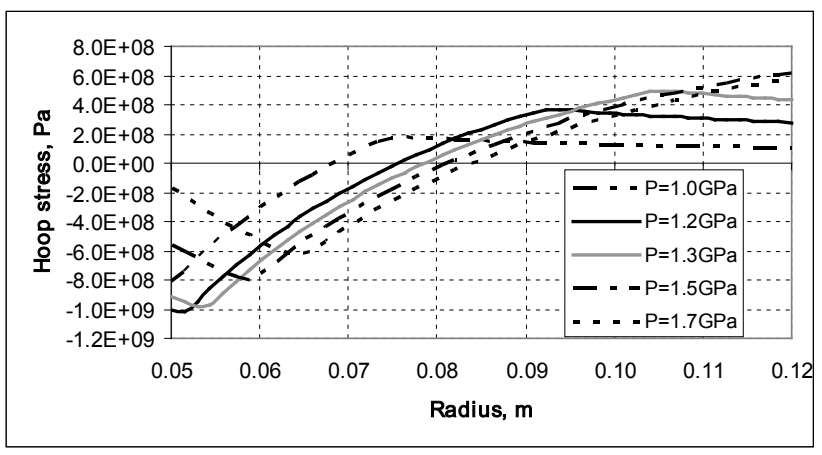

Figure 5. Residual stresses

Influence of damage on residual stress has complex mode. Compressive stress modulus is decreased as a result of damage on the cylinder bore. The maximum of residual hoop stress is displaced from an internal radius.

It is known $[5,6,9]$ that the Bauschinger effect also has influence on distribution of residual stress after autofrettage. Such influencing shows up at the large values of autofrettage pressure because for the appearance of Bauschinger effect initiation of negative plastic strain rates is necessary at unloading. For illustration of such influence the series of calculations are conducted for autofrettage process at the different values of $\mathrm{P}_{\max }$ without taking into account damage. Hoop residual stresses profiles are represented for the autofrettage processes with the single loading to $\mathrm{P}_{\max }$ and 
total unloading (Figure 4).

The analysis of results shows that the influence of Bauschinger effect in this example is begun with the pressure size $\mathrm{P}_{\max }=1.2 \mathrm{GPa}$. For processes with pressures $\mathrm{P}_{\max }>1.2 \mathrm{GPa}$ areas appear with further yielding during removal of the autofrettage pressure. In these areas behavior of hoop material damage profiles changes. Absolute value hoop stress is increased at moving off the bore and have a local maximum on the border of further yielding area.

\section{Conclusion}

On the basis of continuum damage mechanics a mathematical model is derived for determination of residual stress in a solid body after plastic deformation. Investigation of the damage influence is important for special technological presses that has goal to introduce advantageous residual stresses and increase fatigue lifetimes of construction units. Material damage reduces the advantageous residual stress values. In addition, damage accumulated at plastic deformation is initial condition for low or high cycle fatigue analysis by damage mechanics approach.

On example of the autofrettaged thick-walled tube the damage influence was calculated by finite element method. The numerical procedure was used to compare residual hoop stress values with and without the material damage influence.

At planning of autofrettage process conditions the optimizing behavior results in the increase of level of pressure and sizes of plastic strain at the end of loading. But because of material damage residual hoop stress decrease and develop some micro cavities in the material. Similar contradiction arises up from the influence of Bauschinger effect. The mathematical modeling and numerical analysis is needed for finding of compromise between these tendencies.

\section{REFERENCES}

[1] R. Hill. The mathematical theory of plasticity, Oxford University Press, London; 1950.

[2] J. Chakrabarty. Theory of plasticity, McGraw-Hill; New York, 1987.

[3] P. Livieri, P. Lazzarin. Autofrettaged cylindrical vessels and Bauschinger effect: an analytical frame for evaluating residual stress distributions, Journal of Pressure Vessel Technology, Vol.124, No.1, 38-46, 2002.

[4] A. Loghman, M.A. Wahab. Loading and unloading of thick-walled cylindrical pressure vessels of strain-hardening material, Journal of Pressure Vessel Technology, Vol.116, 105-109, 1994.
[5] A.P. Parker, J.H. Underwood, D.P. Kendall. Bauschinger effect design procedures for autofrettaged tubes including material removal and Sachs' method, Journal of Pressure Vessel Technology, Vol.121, 430-471, 1999.

[6] J.H. Underwood, A.P. Parker, E. Troiano. Effect of Bauschinger-modified autofrettage residual stresses on cannon fatigue life, Journal of ASTM International (JAI), Vol.3, No.8, 2006.

[7] J. Bauschinger. Ueber die Veranderung der Elastizitätsgrenze und der Elastizitätsmodulus verschiedener Metalle, Zivilingenieur; Vol.27, 289-348, 1881.

[8] A.P. Parker. Bauschinger effect design procedures for compound tubes containing an autofrettaged layer, Journal of Pressure Vessel Technology, Vol.123, 203-206, 2001.

[9] A. Parker. Autofrettage of open-end tubes - pressures, stresses, strains, and code comparisons, Journal of Pressure Vessel Technology, Vol.123, No.3, 271-281, 2000.

[10] G. Lvov, S. Lysenko. Contact problem for the autofrettage of thick cylinders, Journal of Computational and Applied Mechanics, Vol.3, No.1, 51-60, 2002

[11] W.S. Shim, J.H. Kim, Y.S. Lee, K.U. Cha, S.K. Hong. A study on hydraulic autofrettage of thick-walled cylinders incorporating Bauschinger effect, Experimental Mechanics an International Journal, Vol.50, 621-626, 2010.

[12] X.P. Huang. A general autofrettage model of a thick-walled cylinder based on tensile-compressive stress-strain curve of a material, The Journal of Strain Analysis for Engineering Design, Vol.40, No.6, 599-607, 2005.

[13] Hiroto Okuno, Hideo Koeda, Teruo Takahashi, Hirofumi Tada. Optimization of autofrettage method for thick-walled pressure vessel by using FEM analysis, Nippon Kikai Gakkai Hokkaido Shibu Koenkai Koen Galyoshu, Vol.42, 184-185, 2002.

[14] Ayob Amran, Elbasheer M. Kabashi. Optimum autofrettage pressure in thick cylinders. Jurnal Mekanikal, Department of Applied Mechanics, Faculty of Mechanical Engineering, University Technology, Malaysia, Vol.24, 1-14, 2007.

[15] A.B. Ayob, M.N. Tamin, Elbasheer M. Kabashi. Pressure limits of thick-walled cylinders, Proceedings of the International MultiConference of Engineers and Computer Scientists (IMECS) 2009, Hong Kong, March 18-20, Vol.2, 2009.

[16] M.H. Hojjati, A. Hassani. Theoretical and finite-element modeling of autofrettage process in strain-hardening thick-walled cylinders, International Journal of Pressure Vessels and Piping, Vol.84, 310-319, 2007.

[17] M. Perl, J. Perry. The beneficial contribution of realistic autofrettage to the load-carrying capacity of thick-walled spherical pressure vessels, Journal of pressure vessel technology, Vol.132, No.1, 2010.

[18] P. Parker. A re-autofrettage procedure for mitigation of Bauschinger effect in thick cylinders, Journal of pressure vessel technology, Vol. 126, No.4, 451-454, 2004.

[19] J. Lamaitre. A course on damage mechanics, Verlag Berlin Heidelberg, Springer, 2005.

[20] W. Jiang. The elastic-plastic analysis of tubes. 1. General theory. 2. Variable loading. 3. Shakedown analysis. 4. Thermal ratcheting, Journal of pressure vessel technology, Vol.114, No.2, 213-245, 1992. 